\title{
Correlation between Activated Clotting Time and Activated Partial Thromboplastin Time During Endovascular Treatment of Cerebral Aneurysms
}

\author{
David Faraoni' ${ }^{1}$ Benjamin Mine ${ }^{2}$, Wael Nabhan ${ }^{3}$, Pierre Nokerman $^{3}$, Yvon Deryck ${ }^{3}$, Michel Baurain $^{3}$, \\ Boris Lubicz ${ }^{2}$ \\ ${ }^{1}$ Department of Anesthesiology, Centre Hospitalier Universitaire Brugmann-HUDERF, Université Libre de Bruxelles, Brussels, \\ Belgium; ${ }^{2}$ Department of Anesthesiology, Erasme University Hospital, Université Libre de Bruxelles, Brussels, Belgium; ${ }^{3}$ Depart- \\ ment of Diagnostic \& Interventional Neuroradiology, Erasme University Hospital, Université Libre de Bruxelles, Brussels, Belgium. \\ Email: David.FARAONI@huderf.be
}

Received November $15^{\text {th }}, 2011$; revised December $17^{\text {th }}, 2011$; accepted December $26^{\text {th }}, 2011$

\begin{abstract}
Purpose: Endovascular treatment (EVT) of intracranial aneurysms (IA) requires a continuous anticoagulation to avoid thromboembolic complications. In order to monitor the anticoagulation, different tests may be used including the activated clotting time (ACT) and the activated partial thromboplastin time (APTT). The aim of this study was to compare ACT and APTT for the monitoring of the anticoagulation during EVT of IA. Methods: Patients referred for EVT of an IA were included. After induction, baseline ACT and APTT were recorded, followed by a bolus infusion of unfractionated heparin $\left(50 \mathrm{UI} \cdot \mathrm{kg}^{-1}\right)$. The same tests were controlled five minutes later with the purpose of doubling the baseline ACT value. Correlation and agreement between both tests were evaluated for the percentage of change after the bolus. Multiple linear regressions were also calculated in order to show confounding factors. Complications and outcomes were also recorded. Results: 45 patients were checked for enrolment and 24 were included for analysis. Mean (SD) \% variation for APTT was 432.1 (75.7) and 60.6 (23.0) for ACT with $\mathrm{p}<0.0001$. With the Bland-Altman method, value of Bias (SD) is 372 (86) with 95\% limits of agreement range from 203 to 540 . Pearson correlation for \% variation shows $\mathrm{r}(95 \% \mathrm{CI})=-0.23(-0.58$ to 0.19$)$ with $\mathrm{p}=0.29$ and $\mathrm{R}$ square $=0.05 .100 \%$ of the APTT values could be defined as excessive anticoagulation by opposition of the $8 \%$ obtained with ACT. Conclusions: This prospective observational study shows that ACT test is not well correlated with APTT and leads to a systematic excessive coagulation during EVT of IA.
\end{abstract}

Keywords: Interventional Neuroradiology; Anticoagulation; Monitoring

\section{Introduction}

Treatment of IA has significantly evolved during the last decade [1]. Nowadays, EVT is the first intention treatment of IA in most cases. The endovascular approach requires a precise and continuous anticoagulation in order to avoid thromboembolic complications [2]. Until now, no definitive guidelines have been formulated concerning the optimal anticoagulation regimen during these procedures [3]. Unfractionated Heparin (UFH) is generally used because of the possible titration and because it can be reversed with the administration of protamine sulphate.

In order to monitor the anticoagulation during EVT, different tests may be used including the activated clotting time (ACT) and the activated partial thromboplastin time (APTT).
Even if, APTT appears very sensitive for systemic heparinization monitoring, the delay required to obtain results are too long for a perioperative monitoring [4]. On the other hand, the ACT has been used for several decades in cardiac surgery as well as other procedures, and now in interventional neuroradiology [5-7]. Some controversies remain about the correlation between the different ACT monitoring devices and APTT [8]. The aim of this study was to compare ACT and APTT for the monitoring of the anticoagulation during EVT of IA. This population offer the advantage that the underlying condition does not influence coagulation status.

\section{Materials and Methods}

Our study has been approved by our local ethical committee (Erasme University Hospital Ethic comity refer- 
ence: P2011/117, EudraCT/CCB: B406201110951) and informed consent was obtained for all patients. Inclusion criteria were as follows: patients aged $>18$ years old, scheduled to interventional treatment of ruptured or nonruptured IA were enrolled in our prospective observational study. Patients scheduled to surgical procedure (intracranial pressure catheter or aneurysms treatment), with heparin allergy, or history of heparin-induced thrombocytopenia (HIT) were excluded from the study. Finally, patients previously intubated in the intensive care unit (ICU) for neurologic deterioration based on the Glasgow Coma Scale (GCS) were not considered for inclusion. Anaesthesia protocol was left to the discretion of the anaesthetists. Standard monitoring (5 leads-EKG, non invasive pressure, pulse oxymetry), radial artery catheter, urinary catheter, rectal temperature probe were systematically used but central venous catheter was only added in case of ruptured IA. The trachea was intubated. Blood sample were systematically drawn from the arterial line. ACT was obtained from a GEM PCL Plus device (Portable Coagulation Laboratory, Instrumentation Laboratory, Lexington, MA) using a Kaolin-activated ACT test (GEM Test ACT, Instrumentation Laboratory, Lexington, MA). No guideline exists about ACT time and the dose of UFH for systemic anticoagulation during EVT of IA. Our protocol has been chosen according to clinical experience. After induction, baseline values of ACT and APTT were obtained. Immediately afterwards, the patient was given a bolus of $50 \mathrm{UI} \cdot \mathrm{kg}^{-1}$ of UFH. Five minutes later, the same tests were controlled. Our goal was to double the ACT value. If the target ACT value was not reached a second bolus of $20 \mathrm{UI} \cdot \mathrm{kg}^{-1}$ UFH was administered. A continuous infusion $\left(30 \mathrm{UI} \cdot \mathrm{kg}^{-1} \cdot \mathrm{h}^{-1}\right)$ was maintained during the whole procedure. ACT was controlled every 30 minutes during the procedure. At the end of the treatment, continuous infusion of UFH was adjusted according to the recommendation of the principal operator (BL) with an aim to maintain a therapeutic anticoagulation level. The patient was waked up and extubated in order to rapidly explore neurological status. In the ICU, standard and invasive monitoring was followed while APTT was chosen to monitor anticoagulation. The following data were recorded: age, weight, height, previous use of aspirin (AAS), clopidogrel, low molecular weight heparin (LMWH), previous exposition to UFH. Timing and values of baseline ACT and APTT, controlled values after the first bolus, and at the end of the procedure were recorded. Because of the time necessary to receive the APTT value, the anaesthetists were blinded to these results and perioperative anticoagulation management was based only on ACT values. Length of ICU stay and postoperative complications (rebleeding, thrombosis, others) were also noted and analysed.

\section{Statistics}

Continuous variables are presented as mean (SD) or 95\% confidence interval $(95 \% \mathrm{CI})$. Discrete variables are reported as \%. Percentage (\%) variation for ACT and APTT was calculated (ratio between (controlled value baseline) and baseline) and chosen in order to evaluate if the targeted anticoagulation was reached (double). Moreover, a Pearson correlation was realized. The paired $t$ test was used to compare the means of two matched groups, assuming that the distribution of the before-after differences follows a Gaussian distribution. Secondly, the Bland-Altman method was used to assess agreement between \% variation in ACT and \% variation in APTT. Bias (mean difference) represent the systematic error between both method and precision (SD of the bias) is representative of the random error between the tests. The limits of agreement were calculated as bias $\pm 2 \mathrm{SD}$, and defined the range in which $95 \%$ of the differences between the methods were expected to lie. Multiple linear regressions were performed for patient receiving aspirin, clopidogrel and previously exposed to UFH. For all comparisons, a $\mathrm{p}$ value $<0.05$ was considered statistically significant. Excessive anticoagulation threshold was defined as a $\%$ variation of ACT or APTT $>150 \%$. Statistical analysis were performed with Prism 5 for Mac OS (version 5.0; GraphPad software inc. San Diego California USA, www.graphpad.com) and AnalystSoft, StatPlus: mac - statistical analysis program for Mac OS. Version 2009.

\section{Results}

24 patients were included in our analysis. Characteristics of patients analysed are shown in Table 1. Baseline and controlled mean (SD) ACT were respectively, 120.9

Table 1. Patient characteristics and outcomes. Values are expressed as mean (SD) or number (proportion: \%).

\begin{tabular}{lcc}
\hline & $\mathrm{n}=24(\%)$ & SD or proportion \\
\hline Age (year) & 53 & 13 \\
Height (cm) & 165 & 7 \\
Weight (kg) & 69 & 16 \\
Ruptured & 4 & $17 \%$ \\
Aspirin & 7 & $29 \%$ \\
Clopidogrel & 5 & $21 \%$ \\
Previous exposition & 11 & $46 \%$ \\
to heparin & 0 & $0 \%$ \\
Thrombosis & 0 & $0 \%$ \\
Bleeding & 1.04 & 0.2 \\
ICU stay (day) & & \\
\hline
\end{tabular}


(21.2) s. and 190.5 (31.6) s. Related mean (SD) APTT values were $29.0(6.0)$ s. and $149.9(0.2)$ s. Mean (SD) \% variation for APTT was 432.1 (75.7) and 60.6 (23.0) for $\mathrm{ACT}$ with $\mathrm{p}<0.0001$ (Figure 1). Figure 2 shows the results for the Bland-Altman method, value of Bias (SD) is 372 (86) with $95 \%$ limits of agreement range from 203 to 540 . Pearson correlation for $\%$ variation shows $\mathrm{r}(95 \%$ $\mathrm{CI})=-0.23(-0.58$ to 0.19$)$ with $\mathrm{p}=0.29$ and $\mathrm{R}$ square $=$ 0.05 (Figure 3). Table 2 shows the results of the multiple linear regressions for AAS, clopidogrel and previous exposition to UNF, all tests were statistically not signifycant. According to the results obtained after the first bolus, $100 \%$ of the APTT values could be defined as excessive anticoagulation by opposition of the $8 \%$ obtained with ACT. According to these results, the study was interrupted in order to modify our anticoagulation policy and decrease the excessive rate of excessive anticoagulation.

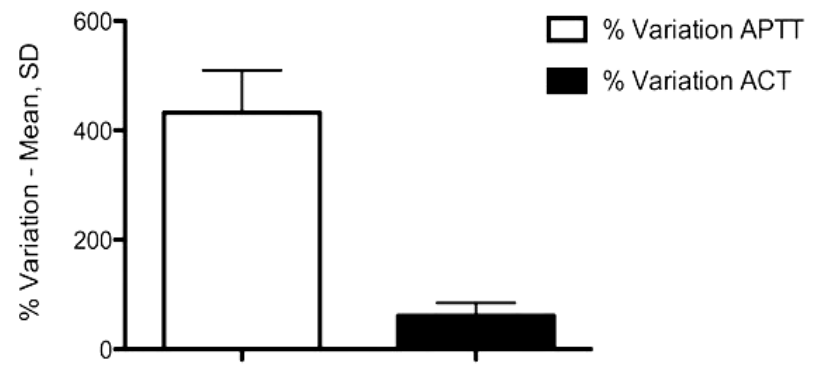

Figure 1. Percentage variation of activated clotting time (ACT) and activated thromboplastin time (APTT) after a bolus of $50 \mathrm{UI} \cdot \mathrm{kg}^{-1}$ of unfractionated heparin. With $\mathrm{p}<$ 0.0001.

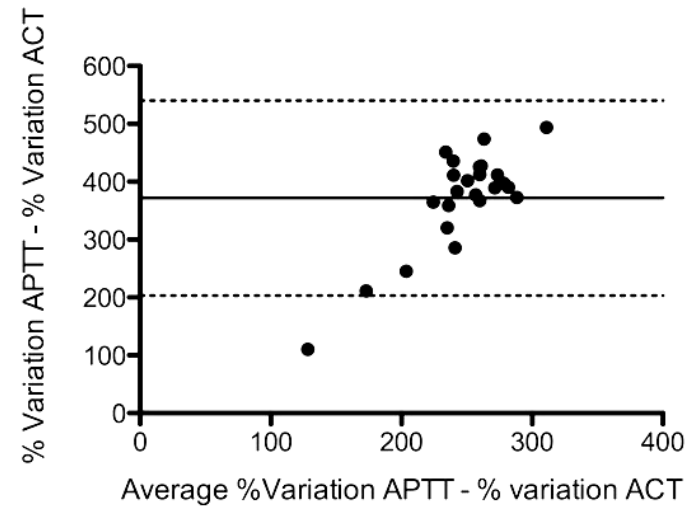

Figure 2. Bland-Altman plots between the percentage of variation in APTT and percentage of variation in ACT after $50 \mathrm{UI} \cdot \mathrm{kg}^{-1}$ of unfractionated heparin. Unbroken lines show the mean difference (bias) and the dotted lines show the 2 SD limits of agreement.

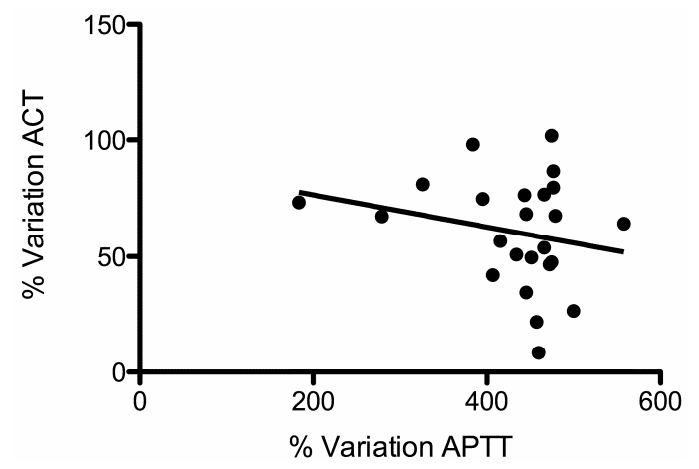

Figure 3. Scatterplot of paired samples $(n=24)$ for the percentage variation of activated clotting time (ACT) and activated partial thromboplastin time (APTT).

Table 2. Multiple linear regressions for aspirin, clopidogrel and previous exposition to unfractioned heparin.

\begin{tabular}{|c|c|c|c|c|c|c|c|}
\hline & Coefficients & $\begin{array}{c}\text { Standard } \\
\text { Error }\end{array}$ & $L C L$ & $U C L$ & t Stat & p-level & $\begin{array}{l}H 0(5 \%) \\
\text { rejected? }\end{array}$ \\
\hline Intercept & 58.96 & 7.32 & 43.69 & 74.23 & 8.05 & 0. & Yes \\
\hline AAS & 11.42 & 11.75 & -13.10 & 35.93 & 0.97 & 0.34 & No \\
\hline Clopidogrel & -11.70 & 13.15 & -39.13 & 15.73 & -0.89 & 0.38 & No \\
\hline Previous UFH & 1.56 & 10.16 & -19.63 & 22.75 & 0.15 & 0.88 & No \\
\hline$T(5 \%)$ & 2.09 & & & & & & \\
\hline
\end{tabular}




\section{Discussion}

Our study shows that ACT monitoring of UFH anticoagulation during interventional neuroradiology is associated with a poor correlation with APTT and especially led to $100 \%$ of excessive anticoagulation.

Aneurysmal subarachnoid haemorrhage ( $\mathrm{SAH})$ is associated with a high rate of neurologic complications such as delayed secondary brain injury, cerebral infarcttion, neuronal death and hydrocephalus, resulting in focal neurologic deficits and cortical dysfunction [9]. In this field, heparin administration has been advocated to reduce complication rate, based on the hypothesis that micro-emboli are an important cause of ischemic injury following SAH treatment [10]. Moreover, a recent study has confirmed the role of heparin to decrease delayed SAH induced neurological deficits [11].

Efficient anticoagulation is also required during endovascular procedures to prevent clot formation and thromboembolic complications in the perioperative period. In a recent study, Schubert et al. show that early heparinization is associated with a lower incidence of micro-embolic signal monitoring [12]. Until now, literature does not provide clear recommendations about best anticoagulation scheme. In general, after obtaining a baseline ACT value, a bolus ranging from 30 to 70 $\mathrm{UI} \cdot \mathrm{kg}^{-1}$ is administered to raise ACT by two or three times [13]. These recommendations are based on clinical experience but have not been evaluated in a large prospective trial.

However, it is important to note that one of the most dangerous complications during the procedure is haemorrhage. Even if it is now widely accepted that endovascular treatment has led to decrease the rate of perioperative haemorrhage, intraoperative haemorrhage occurred however in approximately $7.7 \%$ of IA treated by EVT and in $34.5 \%$ treated by clipping surgery [14]. Haemorrhage is often accompanied by an abrupt rise in arterial pressure and immediate protamine reversal may be required.

For all of these reasons, a precise point-of-care monitoring has to be used in the perioperative period of IA treatment. APTT is frequently used to monitor anticoagulation but a central laboratory usually performs it and results take about 45 minutes. This delay could lead to inadequate anticoagulation and should not be routinely used as a bedside monitoring. ACT measures the time required by whole blood samples to clot after contact activation by either kaolin or celite [15]. Normal values range from $100 \mathrm{~s}$ to $140 \mathrm{~s}$ [16]. The test was first evaluated for cardiac surgery where a high level of anticoagulation is requisite [17] but some controversies persist about its sensitivity for low range (LR) anticoagulation in vascular and intensive care [18].
Our study shows that when used for LR anticoagulation monitoring, ACT leads to an excessive anticoagulation. In our experience, no haemorrhagic complication has occurred. However, such a level of anticoagulation is probably not recommended and could possibly lead to lethal complications. It is important to note that our results are only applicable for GEM PCL Plus device (Portable Coagulation Laboratory, Instrumentation Laboratory, Lexington, MA) using a Kaolin-activated ACT test (GEM Test ACT, Instrumentation Laboratory, Lexington, MA).

Many limitations could be formulated about our study. First, we used ACT designed for high-range heparinization and it could be replaced by an ACT-LR test (GEM Test ACT, Instrumentation Laboratory, Lexington, MA) defined by the manufacturer to be used in case of low range anticoagulation. However the accuracy of the ACT-LR is still subject to discussion. Racioppi et al. have recently compared ACT-LR (Hemochron ${ }^{\circledR}$ jr II Signature device) with APTT and have concluded that the poor correlation between ACT-LR and APTT does not support routine use of this device [19]. The same device was evaluated by Tremey et al. in vascular surgery and was compared to anti-Xa activity [6]. They demonstrated the accuracy of the device, during vascular surgery, with the use of $50 \mathrm{UI} \cdot \mathrm{kg}^{-1} \mathrm{UFH}$. ACT-LR was well correlated with anti-Xa activity. Further studies are needed to evaluate the correlation of GEM Test ACT-LR and anti-Xa activity. Second, Kaolin was used as activetor in our device. According to manufacturer recommenddations, Celite could have been preferred in this case. However, no evidence exists about the difference between Kaolin-activated and Celite-activated probes.

The use of APTT to monitor heparin therapy is not based on randomized controlled clinical trials, and the test is associated with significant intra- and interindividual variability, not related to circulating blood heparin activity. For this reason anti-Xa analysis, which reflects the heparin activity, are now used by many laboratories [20]. Unfortunately, this test could not be used as a bedside monitoring and furthermore it agreement is also discussed in the literature.

Thromboelastography is more and more used for bedside evaluation of coagulation disorders, but this test is not specifically designed to monitor UFH and results are obtained after more than some minutes [21].

A portable bedside point-of-care instrument (CoaguCheck ${ }^{\circledR}$ Pro, Boehringer Mannheim Diagnostics, USA, Roche Diagnostics, Switzerland) allows APTT measurements in three minutes [22]. In a Canadian study, the agreement between point-of-care monitoring and standard laboratory tests was relatively poor [23].

The summary of this discussion appears clear: clini- 
cians do not dispose of an ideal point-of-care monitoring. When chosen, the user needs to know the limitations.

Further studies are needed to evaluate the efficacy of ACT-LR with classical ACT and to define the optimal targeted anticoagulation for interventional neuroradiology.

\section{Conclusion}

In conclusion, our prospective observational study shows that GEM PCL plus HR-ACT test is not well correlated with APTT and leads to a systematic excessive coagulation during EVT of IA. Even if no complications were described in our study, careful evaluation of the anticoagulation is required and further studies are needed to evaluate the best device and the best anticoagulation scheme during treatment of IA. Finally, clinicians need to know the device used and choose their test according to the manufacturer recommendations.

\section{REFERENCES}

[1] M. J. Gounis, M. J. De Leo and A. K. Wakhloo, "Advances in Interventional Neuroradiology," Stroke, Vol. 41, No. 2, 2010, e81-e87. doi:10.1161/STROKEAHA.109.574319

[2] Y. Fujii, S. Takeuchi, T. Koike, K. Nishimaki, Y. Ito, R. Tanaka and K. Okamoto, "Heparin Administration and Monitoring for Neuroangiography," American Journal of Neuroradiology, Vol. 15, No. 1, 1994, pp. 51-54.

[3] A. Bandeira, G. Raphaeli, D. Baleriaux, M. Bruneau, O. De Witte and B. Lubicz, "Selective Embolization of Unruptured Intracranial Aneurysms is Associated with Low Retreatment Rate," Neuroradiology, Vol. 52, No. 2, 2010, pp. 141-146. doi:10.1007/s00234-009-0607-7

[4] H. P. Grill, J. E. Spero and J. E. Granato, "Comparison of Activated Partial Thromboplastin Time to Activated Clotting Time for Adequacy of Heparin Anticoagulation Just before Percutaneous Transluminal Coronary Angioplasty," American Journal of Cardiology, Vol. 71, No. 13, 1993, pp. 1219-1220.

doi:10.1016/0002-9149(93)90649-W

[5] N. A. Guzzetta, H. G. Monitz, J. D. Fernandez, T. M. Fazlollah, A. Knezevic and B. E. Miller, "Correlations between Activated Clotting Time Values and Heparin Concentration Measurements in Young Infants Undergoing Cardiopulmonary Bypass," Anesthesia and Analgesia, Vol. 111, No. 1, 2010, pp. 173-179.

[6] B. Tremey, B. Szekely, S. Schlumberger, D. François, N. Liu, K. Sievert, M. Fischler, “Anticoagulation Monitoring During Vascular Surgery: Accuracy of the Hemochron Low Range Activated Clotting Time (ACT-LR)," British Journal of Anaesthesia, Vol. 96, No. 4, 2006, 453-459. doi:10.1093/bja/ael194

[7] R. Kubalek, A. Berlis, M. Schwab, J. Klisch, M. Schumacher, "Activated Clotting Time or Activated Partial Thromboplastin Time as the Method of Choice for Patients
Undergoing Neuroradiological Intervention," Neuroradiology, Vol. 45, No. 5, 2003, 325-327.

[8] J. J. De Waele, S. Van Cauwenberghe, E. Hoste, D. Benoit, F. Colardyn, "The Use of the Activated Clotting Time for Monitoring Heparin Therapy in Critically Ill Patients," Intensive Care Medicine, Vol. 29, No. 2, 2003, pp. 325-328.

[9] J. B. Bederson, E. S. Connolly Jr., H. H. Batjer, R. G. Dacey, J. E. Dion, M. N. Diringer, J. E. Duldner Jr, R. E. Harbaugh, A. B. Patel and R. H. Rosenwasser, "Guidelines for the Management of Aneurysmal Subarachnoid Hemorrhage: A Statement for Healthcare Professionals from a Special Writing Group of the Stroke Council, American Heart Association," Stroke, Vol. 40, No. 3, 2009, pp. 994-1025. doi:10.1161/STROKEAHA.108.191395

[10] M. D. Vergouwen, M. Vermeulen, B. A. Coert, E. S. Stroes and Y. B. Roos, "Microthrombosis after Aneurysmal Subarachnoid Hemorrhage: An Additional Explanation for Delayed Cerebral Ischemia," Journal of Cerebral Blood Flow and Metabolism, Vol. 28, No. 11, 2008, pp. 1761- 1770. doi:10.1038/jcbfm.2008.74

[11] J. M. Simard, D. Schreibman, E. F. Aldrich, B. Stallmeyer, B. Le, R. F. James and N. Beaty, "Unfractionated Heparin: Multitargeted Therapy for Delayed Neurological Deficits Induced by Subarachnoid Hemorrhage," Neurocritical Care, Vol. 13, No. 3, 2010, pp. 439-449. doi:10.1007/s12028-010-9435-1

[12] G. A. Schubert, C. Thome, M. Seiz, C. Douville and J. Eskridge, "Microembolic Signal Monitoring after Coiling of Unruptured Cerebral Aneurysms an Observational Analysis of 123 Cases," American Journal of Neuroradiology, Vol. 32, No. 8, 2011, pp. 1386-1391.

[13] M. K. Varma, K. Price, V. Jayakrishnan, B. Manickam and G. Kessell, "Anaesthetic Considerations for Interventional Neuroradiology," British Journal of Anaesthesia, Vol. 99, No. 1, 2007, pp. 75-85.doi:10.1093/bja/aem122

[14] S. Suzuki, A. Kurata, M. Yamada, K. Iwamoto, K. Nakahara, K. Sato, J. Niki, M. Sasaki, T. Kitahara, K. Fujii and S. Kan, "Outcomes Analysis of Ruptured Distal Anterior Cerebral Artery Aneurysms Treated by Endosaccular Embolization and Surgical Clipping," Interventional Neuroradiology, Vol. 17, No. 1, 2011, pp. 49-57.

[15] P. G. Hattersley, "Activated Coagulation Time of Whole Blood," Journal of the American Medical Association, Vo. 196, No. 5, 1966, pp. 436-440. doi:10.1001/jama.1966.03100180108036

[16] J. D. Olson, C. F. Arkin, J. T. Brandt, M. T. Cunningham, A. Giles, J. A. Koepke, D. L. Witte, "College of American Pathologists Conference XXXI on Laboratory Monitoring of Anticoagulant Therapy: Laboratory Monitoring of Unfractionated Heparin Therapy," Archives of Pathology and Laboratory Medicine, Vol. 122, No. 9, 1998, pp. 782- 798.

[17] M. T. Ganter, A. Monn, R. Tavakoli, R. Klaghofer, A. Zollinger and C. K. Hofer, "Kaolin-Based Activated Coagulation Time Measured by Sonoclot in Patients Undergoing Cardiopulmonary Bypass," Journal of Cardiotho- 
racic and Vascular Anesthesia, Vol. 21, No. 4, 2007, pp. 524- 528. doi:10.1053/j.jvca.2006.12.012

[18] A. I. O’Neill, C. McAllister, C. F. Corke and J. D. Parkin, "A Comparison of Five Devices for the Bedside Monitoring of Heparin Therapy," Anaesthesia and Intensive Care Journal, Vol. 19, No. 4, 1991, pp. 592-596.

[19] L. Racioppi, A. Quinart, M. Biais, K. Nouette-Gaulain, P. Revel and F. Sztark, "Validation of a Bedside Activated Clotting Time Test (Hemochron Jr II Signature) with Low Dose Heparin Therapy," Anaesthesia, Vol. 64, No. 4, 2009, pp. 430-434.

[20] A. Cuker, B. Ptashkin, B. A. Konkle, S. W. Pipe, H. C. Whinna, X. L. Zheng, D. B. Cines and E. S. Pollak, "Interlaboratory Agreement in the Monitoring of Unfractionated Heparin Using the Anti-Factor Xa-Correlated Activated Partial Thromboplastin Time," Journal of Thrombosis and Haemostasis, Vol. 7, No. 1, 2009, pp. 80-86. doi:10.1111/j.1538-7836.2008.03224.X
[21] D. Bischof, "Thrombelastography in the Surgical Patient," Minerva Anestesiologica, Vol. 76, No. 2, 2010, pp. 131- 137.

[22] K. Ruzicka, S. Kapiotis, P. Quehenberger, S. Handler, S. Hornykewycz, A. Michitsch, K. Huber, D. Clemens, M. Susan, I. Pabinger, S. Eichinger, B. Jilma and W. Speiser, "Evaluation of Bedside Prothrombin Time and Activated Partial Thromboplastin Time Measurement by Coagulation Analyzer CoaguCheck Plus in Various Clinical Settings," Thrombosis Research, Vol. 87, No. 5, 1997, pp. 431-440. doi:10.1016/S0049-3848(97)00159-X

[23] M. Ferring, G. Reber, P. de Moerloose, P. Merlani, M. Diby, B. Ricou, "Point of Care and Central Laboratory Determinations of the aPTT Are not Interchangeable in Surgical Intensive Care Patients," Canadian Journal of Anaesthesia, Vol. 48, No. 11, 2001, pp. 1155-1160. doi:10.1007/BF03020384 